\title{
Tuberculosis of Spine Magnetic Resonance Imaging (MRI) Evaluation of 42 Cases
}

\author{
Khalequzzaman S1, Hoque HW2
}

\begin{abstract}
Tuberculosis of spine is common for Bangladesh. Imaging modality is importent for diagnosis of it.Forty-Six clinically suspected tubeculous spondylitis patient were included in this prospective, consecutive study during July 2003 to June 2005. Among them, 42 cases of MRI and histology proven were included in the article. The peak incidence was found to be in 3 rd decade (43.48\%) with male predominance, 2.5 times more than female. The mean age was revealed 33.3 years. Highest occurrence was in double vertebrae involvement (42.86\%) along with continuous vertebral involvement (85.71\%). Destruction \& collapse found in most case (88.10\%) with predominance with posterior element involved (54.76\%). Spinal deformity was least (11.90\%). Para-spinal soft tissue involvement was found in most cases ((80.95\%) with no calcification. $21.43 \%$ shows cord compression. MRI was found sensitive and accurate modality for diagnosis of TB spondylitis.
\end{abstract}

\section{Introduction}

Percival Pott first described spine tuberculosis classically in $1778^{1}$. Spine tuberculosis is the commonest form of skeletal tuberculosis and constitutes about 50 percent of all cases of tuberculosis of bones and joints. Tuberculosis spondylitis now accounts for 6 percent of new extrapulmonary tuberculous cases $^{1}$. In developing countries, tuberculous spondylitis is a disease of children, whereas in North America and Europe it is most prevalent in middle-aged adults. The disease is equally distributed between both sexes ${ }^{2}$. However, Dharmalingam (2004) showed that the mean age of diagnosis of tuberculous spondylitis was 36.5 and peak incidence is in the second decade of life $(27.3 \%)^{3}$. There were 24 males and 9 females. The majority of the lesions involved the thoracic spine (30.3\%), followed by the lumbar spine (27.2\%). Skip lesions was seen in $12.1 \%$ of cases. Concomitant tuberculosis of the lung was $66.6 \%{ }^{3}$. The order of frequency in Paus (1964) series of 141 cases has been Lumbar (50), dorsal (35), dorsolumbar (25), lumbosacral (22), cervicodorsal (8), sacral (1) and cervical $(\text { nil })^{4}$. Isolated solitary vertebral body tuberculosis is seen in only $1.69 \%$ of the total proven cases of spine tuberculosis 5 . However, Paraspinous abscesses are present in 55 percent to 95 percents of cases $^{6}$. Tuberculosis can affect different parts of vertebra. In a study where 122 cases were studied, the lesion involved one location in 98 cases, two localizations in 9 cases and multiple localizations in 15 cases. The localizations were: posterior arch (20 cases), Centro somatic (10 cases), sub occipital (4 cases) and subligamentous (2 cases) (9). Neurological involvement was seen in $51.5 \%$ of patients ${ }^{3}$. MR

1. Correponding Author: Syed Khalequezzaman MBBS, M.Phil, MD Registrar, Radiology Department, Apollo Hospital, Dhaka skhalequezzaman@apollodhaka.com

2. Hasnat Waheedul Hoque MBBS, M.Phil Assistant Professor of Radiology Cox'sbazar Medical College, Cox'sbazar imaging is the modality of choice for the detection, staging, and differential diagnosis of inflammatory disorders of the spine ${ }^{5}$. It allows the correct diagnosis to be made in all cases, demonstrating the pathological involvement of the Para vertebral structures and into spinal canal earlier and more accurately than CT and plain radiography ${ }^{7,8}$ MRI Imaging of spinal infections requires the use of a combination of T1weighted and T2-weighted or STIR sequences. MRI scans invariably show loss of cortical definition of the affected vertebrae. However, affected vertebrae are often at least partially maintained in pyogenic spondylitis. T1WI often shows infection spread beneath the longitudinal ligaments to involve adjacent vertebral bodies. The discs are sometimes relatively spared, particularly in relationship to the degree of bone destruction ${ }^{9}$. Contrast enhancement is useful and helps to define Para spinal and epidural disease. The aim and objective of the study is to find age and gender incidence among our population and regional distribution in the spinal column and involvement of number of vertebral involvement.

\section{Materials and Methods}

This cross-sectional study was carried out in the department of Radiology and Imaging, BSMMU and in the in-patient department of Centre for the Rehabilitation of the Paralyzed, Savar, Dhaka from July 2003 to June 2005. Forty six patients appeared as tuberculous spondylitis on clinical and X-ray findings were included in the study. MRI was done and peroperative biopsy was taken from each patient and 42 of them were confirmed as the patients of spine TB by both procedures.

MRI examination of spine was routinely performed in the sagittal and axial planes. Sagittal images are obtained with T1 and $\mathrm{T} 2$ weighted images by using spin-echo and fast spin-echo technique. Axial images were obtained with T1 weighted spin echo sequence and proton density weighted. Comparison of T1 and T2 weighted images was one of the fundamental principles of image interpretation. Intravenous gadolinium DTPA MR contrast injection improves MR sensitivity for intramedullary, intra-dural and extra medullary neoplasm and inflammatory process.A summary of the techniques utilized for MR imaging of the spine.

Cervical spine: T1 SAG/AP, T2 FSE(96) SAG H/F, T1 TRA, T2 FSE (16/112) TRA.

Dorsal spine: T1 SAG T2 FSE (96) SAG, T1 TRA, T2 (W-F) TRA.

Lumbar spine: T1sag, T2FSE(8) SAG, T1 TRA, T2 FSE(16/112) TRA.

After contrast: T1 SAG, T1 TRA. T1 FS AXIAL

The aims and objectives of the study were explained to the patients. It was assured that all information would keep secret and procedure would not harm to the patient. Relevant data for each patient were collected and recorded in pre-designed 
individual data collection sheet. All relevant collected data were compiled and analyzed by SPSS. Standard statistical formula ['Z'test ] was applied for significant test. Results Forty six (46) patients were selected from 11 to 69 years of age. The mean age was $33.33 \pm 5.23$ years. It was evident that highest affected individuals (43.48\%) were between 31-40 years of age followed by $21.74 \%$ in age range from $41-50$ years. Older age group (61-70) rather least affected (4.35\%) ( Table-1).

\begin{tabular}{|lcc|}
\hline Age group & Frequency & Percentage \\
\hline $11-20$ & 5 & 11.9 \\
\hline $21-30$ & 6 & 14.3 \\
\hline $31-40$ & 18 & 42.9 \\
\hline $41-50$ & 8 & 19.0 \\
\hline $51-60$ & 3 & 7.1 \\
\hline $61-70$ & 2 & 4.8 \\
\hline Total & $\mathbf{4 2}$ & $\mathbf{1 0 0 . 0}$ \\
\hline
\end{tabular}

In our series 33 (71\%) were male and 13 (29\%) were female). The male female ratio was $2.5: 1$ and highly statistically significant $(\mathrm{P}<0.001)$ ( Figure-1).

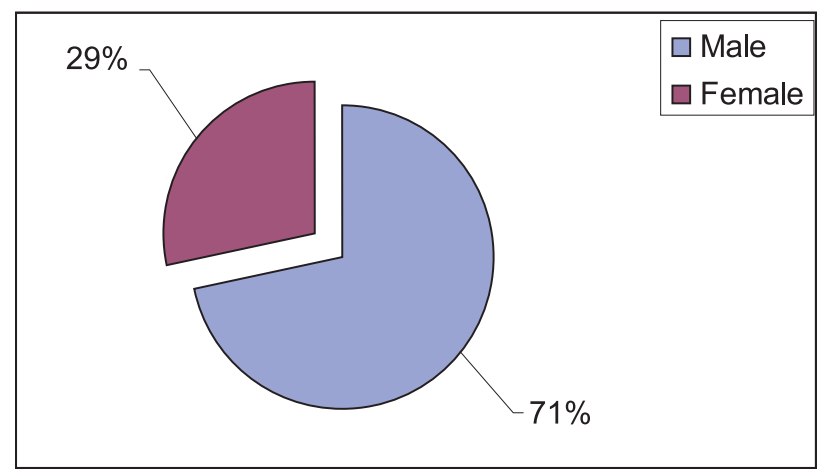

Figure: 1 Sex distribution of patients

Five $(10.8 \%)$ were neck pain, $65.2 \%$ were low back ache. Forty two $(91.3 \%)$ had functional disability, $41.3 \%$ had fever and $10.8 \%$ had deformity. None was experienced radiculopathy. MRI shows regional vertebral involvement. Five (10.8\%) had cervical involvement, $6.5 \%$ had upper thoracic involvement is noted. In $8.7 \%$ cases lower thoracic vertebra \& thoraco-lumbar vertebra, in $19.6 \%$ cases lumbar was involved ( Fig-2 a). No sacral piece was involved.

Vertebrae was involved- single $3(7.14 \%)$, two $18(42.86$, three $14(33.33 \%)$ more than three $7(16.67 \%)$, continuous $36(85.71 \%)$, non continuous $6(14.29 \%)$, destruction \& collapse $37(88.10 \%)$, none $5(11.90 \%)$, posterior element involved $23(54.76 \%)$, non involved $19(45.24 \%)$, intraosseous abscess $15(35.71 \%)$, Signal change $42(100.0 \%)$, and spinal deformity $5(11.90 \%)$ (Fig- 2 b\& c).

In soft tissue it was found that epidural was $23(54.76 \%)$, paraspinal 34(80.95\%), subligamentous 33(78.57\%), calcification $0(0.0 \%)$, Myelitis $4(9.52 \%)$ and Cord compression $9(21.43 \%)$. In disc it was observed that thinning was $31(73.81 \%)$, destruction $28(66.67 \%)$, endplate changes $30(71.43 \%)$ and single changes $30(71.43 \%)$.
Here $40(83.33 \%)$ cases of tuberculous spondylitis were diagnosed correctly, $1(2.08 \%)$ patient of without tuberculous spondylitis diagnosed wrongly as tuberculous spondylitis. Again 3(6.25\%) cases were correctly diagnosed as not tuberculous spondylitis, $2(4.17 \%)$ cases were wrongly interpreted as not tuberculous spondylitis that had actually had tuberculous spondylitis. One $(2.08 \%)$ false positive case had wrongly diagnosed as tuberculous spondylitis, but actually was metastatic deposit. 2 (4.17\%) false negative cases, which were patient of tuberculous spondylitis, but diagnosed as secondaries in the spine, because of involvement of pedicles. Here sensitivity and specificity of MRI were $95.2 \%$ and $75 \%$ respectively. Here accuracy of MRI was $93.5 \%$. (Table-II).
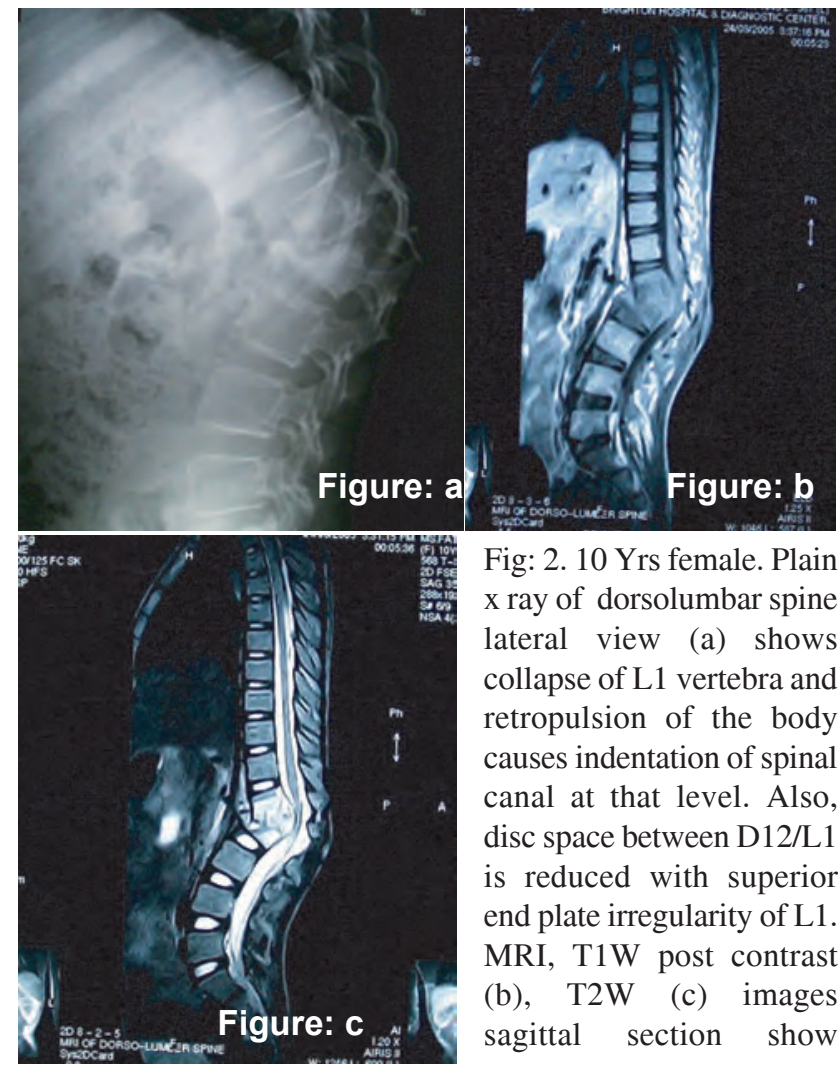

Fig: 2. 10 Yrs female. Plain $\mathrm{x}$ ray of dorsolumbar spine lateral view (a) shows collapse of L1 vertebra and retropulsion of the body causes indentation of spinal canal at that level. Also, disc space between D12/L1 is reduced with superior end plate irregularity of L1. MRI, T1W post contrast (b), T2W (c) images sagittal section show

heterogeneously enhancing intra-osseous and epidural mass, causing severe indentation at the conus medullaris associated with myelopathy. It shows destruction of disc between D12/L1 and pr evertebral soft tissue mass.

\section{Discussion}

Tuberculous spondylitis can occur in any age. Middle aged adults are the most frequently affected by tuberculous spinal infection ${ }^{10}$. In my study, the most common age presenting with tuberculous spinal infection is between 31 to 40 years (43\%), which was less than the presentation in the developed countries. Because, lot of risk factors took place for the development of tuberculosis, like, low nutrition, overcrowding, poor hygienic condition, multipariety etc. In western and developed countries, tuberculosis was a delayed presentation as because, only risk factor which play important role for the development of this disease is immucompromised states like diabetes, old age and more recently AIDS.

Males suffer more than females ${ }^{11}$. In my study also showed male were more sufferer than females, about 2.5 times more than females. 
In my study, more than $50 \%$ of cases involve lumbar vertebra, which is not differed from the observation by TK Loke ${ }^{12}$. In same series, single vertebral body involvement found only $7 \%$ of cases. The results of my study are near to that result $(5 \%)^{12}$. However, Lolge showed fewer cases (1.69\%) of solitary vertebral

body involvement ${ }^{5}$. Single vertebral body involvement may mimic metastatic involvement. It is likely that single vertebral body involvement represents an early stage of disease and, in my experience, most cases will present with advanced disease due to its insidious onset. Involvement of three or more vertebral bodies was found in $50 \%$ of my cases, more frequent than in other series (14-22\%) carried out by Smith ${ }^{6}$. This reflects advanced disease and the route of transmission due to hematogeneous and subligamentous spread ${ }^{13}$. The multiplicity of vertebral body involvement may cause confusion with metastatic disease. Skip lesions located at the cervical spine have been reported in as many as $12 \%$ of cases $^{13}$. There may be a need to exclude non contiguous lesions in the lower spine in those patients with cervical spondylitis, especially when low back symptoms are present.

Almost all TB spondylitis will show some form of vertebral body destruction. In my studies, it is $88 \%$. Loke performed a study which showed vertebral body destruction were $73 \%$, slightly lower that I found in my study $(88 \%)^{12}$. In my experience, axial MR images will demonstrate cortical destruction and posterior element involvement to best advantage. Posterior element involvement was found in 55\% which is slightly more incidence than that found in other reported series ${ }^{12}$, even though it has been reported more frequent in non white population ${ }^{14,15}$. There is a tendency towards pedicular and laminar involvement in tuberculous spondylitis, whereas pyogenic spondylitis has a predilection for the facet joint ${ }^{14}$. The presence of posterior element involvement is a significant finding, since these patients are more likely to have neurological symptoms and require laminectomy ${ }^{6}$. Bilateral pedicle involvement is infrequent, but it results in an unstable spine and often necessitates a different approach ${ }^{16}$. Posterior element involvement is frequently seen as an extension of the vertebral body disease, but Babhulkar et al showed that isolated diseases of posterior element in TB spondylitis have a reported incidence ranging from 0.8 to $10 \%{ }^{17}$. These cases of posterior element abnormalities without vertebral body involvement make differentiation of an infection from a tumor very difficult.

An abnormal vertebral body signal is invariably seen. There is a confluently decrease signal of vertebral body and associated interspace, with poor distinction between these on short TR/TE images. There is often an increased signal intensity of the vertebral body adjacent to the involved disc on T2 weighted images. Similar to other spinal infections, an increased signal on T1 weighted images from previously affected vertebrae indicates healing and has been found to correlate well with clinical signs and symptoms ${ }^{18}$. Signal change with hyperintensity on short TR/TE that is seen with healed or old TB is due to infiltration of hemopoietic marrow with fat, and also been reported to occur as early as 1 month, making MRI potentially very useful for monitoring the effects of chemotherapy.

Most active disease has paraspinal (subligamentous, psoas, epidural) soft tissue masses. Magnetic resonance imaging is particularly useful in demonstrating the morphologic extent of soft tissue spread, especially after GdDTPA injection. Paraspinal abscesses are often bilateral, large and relatively out of proportion to the amount of bone destruction ${ }^{13,19,20}$.

Psoas abscesses occur below the level of spondylitis. Small calcifications characteristic of TB may be undetected on MRI, but the presence of other imaging findings such as subligamentous spread, intra osseous and paraspinal abscesses with thick rim enhancement is diagnostic. Subligamentous spread, paraspinal extension and the development of psoas abscesses are common findings. Enhanced coronal images are extremely useful for delineating these abscesses and their communications.

Gadolinium MRI is particularly useful for characterizing TB spondylitis ${ }^{10,18}$. Thick rib enhancement around intra osseous and paraspinal soft tissue abscesses has not been demonstrated in other spinal infections ${ }^{18}$. Gero found that epidural extension and intradural disease (meningitis and myelitis) are seen to better advantage on enhanced $\operatorname{scan}^{21}$. The presence of reactivation, abscesses versus cellulitis, is often diagnosed with confidence only after GdDTPA enhancement. Both abscesses and cellulitis show similar signal intensities on unenhanced $\mathrm{T} 1$ and $\mathrm{T} 2$ weighted images, and their differentiation is important because abscesses require drainage. Post suggested that in pyogenic spondylitis, dense enhancement of vertebral body with GdDTPA enhancement in MR imaging appears to indicate an active infection, whereas minimal or negligible contrast medium uptake is indicative of resolving infection ${ }^{22}$ It remains to be seen whether the same is true for $\mathrm{TB}$ spondylitis. Unlike pyogenic infection, it has been reported that disc involvement in TB spondylitis is uncommon. Smith (1989) and Corr (1991) reported an incidence of $25 \%$ of patients with abnormal signal intensities of the disc on T2 weighted images, a finding commonly seen in pyogenic spondylitis ${ }^{6,23}$. This difference has been attributed to a lack of proteolytic enzymes in the mycobacterium, leading to a relative preservation of the intervertebral disc ${ }^{6}$. Non involvement of the disc on pathological examination has been reported more often in non whites ${ }^{14,15}$. This has not been case in my series, with more than $73 \%$ of cases showing some form of disc abnormalities. This discrepancy may be due to the high incidence of advanced disease in this locality, because disc involvement occurs secondarily, with spread of infection beginning at the antero-inferior portion of the vertebral body Discitis appears as disc thinning but it has a high T2 signal (although a type 1 modic degenerative disc may also show an elevated T2 signal). There is an abnormal increased signal of the disc on T2 weighted images, with associated hyperintensities of the end plates at the abnormal disc level.

Magnetic resonance imaging is useful in the detection of the reactivation of old $\mathrm{TB}$ spondylitis. The presence of active disease is demonstrated as vertebral body destruction, especially with concomitant paraspinal soft tissue and/or intra osseous abscesses. It has been reported that thick rim enhancement in these abscesses is strongly suggestive of tuberculous infection and is not seen in non granulomatous spondylitis ${ }^{18}$. The change of signal intensity from low signal in healed TB to high signal on T2 weighted images suggests active disease. Vertebral body and disc enhancement may be 
seen after intravenous contrast.

MRI should be considered to be the imaging modality of choice for patients with suspected TB spondylitis, because of its high specificity, sensitivity and accuracy as it provides necessary information to the surgeon for proper management by providing vertibral intra-osseous abscess, vertibral disc collapse, skip lesions, dural and intradural diseases and involvement of posterior elements more precisely.

\section{Acknowledgement}

Social welfare department of CRP, Dhaka for partial support in this research.

\section{Reference}

1. Thrush A., Enzmann D. MR Imaging of infectious spondylitis, AJNR 1990; 11:1171-1180.

2. Tuli SM (Editor). Tuberculosis of the skeletal systems. 2en ed. Delhi: Jaypee 1997;5-14.

3. Dharmalingam M. Tuberculosis of the spine-Sabah exterience. Epidemiology, treatment and results, Tuberculosis 2004; 84:24-28.

4. Paus P. Treatment for tuberculosis of the spine, ACTA Orthop (Suppl) 1964;72.

5. Lolge S., Maheshwari M., Shah J., Patkar D., Chawla A. Isolated solitary vertebral body tuberculosis-study of seven cases, Clin Radiol 2003; 58:545-50.

6. Smith AS, Weinstein MA, Mizushima A, Coughlin B, Hayden SP et al. MR imaging characteristics of luberculous spondylitis vs vertebral osteomyclitis, AJR 1989;153:399-405.

7. Ridley N., Shaikh MI., Remedios D., Mitchell R. Radiology of skeletal tuberculosis, Orthopaedics 1998; 21:1213-1220.

8. Zamiati W., Jiddane M., el Hassani MR., Chakir N., Boukhrissi N. Contribution of spiral CT and MRI in spinal tuberculosis, J Neuroradiol 1999; 26:27-34.

9. Osborn AG. (Editor). Diagnostic Neuroradiology. 1st ed. Missouri: Mosby: 1994;822-824.

10. Sharif HS. Role of MR imaging in the management of the spinal infections, AJR 1992;158:1333-1345.
11. Liu GC., Chou MS., Tsai TC, Lin SY., Shen YS. MR evaluation of tuberculous spondylitis, Acta Radiol 1993;34:554-8.Desai SS. Early diagnosis of spinal tuberculosis by MRI, J Bone Joint Surg Br 1994 ;76:863-9.

12. Loke TK., Ma HTG., Chan CS. Magnetic Resonance Imaging of the tuberculous spinal infection, Australas Radiol 1997;4:7-12.

13. Weaver P., Lifeso RM. The radiological diagnosis of tuberculosis of the adult spine, Skeletal Radiology 1984; $12: 178$.

14. Chapman M., Murray RO., Stoker DJ. Tuberculosis of the bones and joints, Semin. Roentgenol 1979; 14:266-82

15. Naim Ur-Rahman. Atypical forms of spinal tuberculosis, J. Bone Joint Surg 1980; 62;162-5.

16. Travlos J., du Toit G. Spinal tuberculosis : Beware the posterior elements, J. Bone Joint Surg 1990;72: 722-3.

17. Babhulkar SS., Tayade WB., Babhulkar SK. Atypical spinal tuberculosis, J. Bone Joint Surg 1984;66:239-42.

18. Sharif HS., Clark DC., Aabed MY., Haddad MC., Al Deeb SM et al. Granulomatous spinal infections: MR imaging, Radiology 1990;177:101-107

19. Whelan MA., Naidich DP., Post JD., Chase NE. Computed tomography of spinal tuberculosis, J. Comput. Assist. Tomogr 1983; 7:25-30.

20. LaBerge JM., Brant Zawadzki M. Evaluation of Pott's disease with computed tomography, Neuroradiology 1984;26:429-434.

21. Gero B., Sze G., Sharif H. MR imaging of intradural inflammatory diseases of the spine, AJNR 1991;12:100919.

22. Post MJD., Sze G., Quencer RM., Eismont FJ., Green BA., Gahbauer H. Gadolinium-enhanced MR in spinal infection, J. Comput. Assist. Tomogr 1990; 14:721-9.

23. Naim-ur-rahman, Jamjoom A, Jamjoom ZA, Al-Tahan AM. Neural arch tubrculosis: radiological features and their corellation with surgical findings, $\mathrm{Br} \mathrm{j}$ Neurosurger. $1997 ; 11: 32-8$ 